\title{
Multiparametric MRI and 18F-DCFPyL PET/CT for Detection of Locally Recurrent Prostate Cancer After Low dose Rate Brachytherapy
}

\author{
Stephanie M. Walker \\ National Cancer Institute \\ Martina Fernandez \\ National Cancer Institute \\ Soumyajit Roy \\ National Cancer Institute \\ Esther Mena \\ National Cancer Institute \\ Jonathan Sackett \\ National Cancer Institute \\ Ronnie Mease \\ Johns Hopkins University School of Medicine

\section{Maria Merino} \\ National Cancer Institute \\ Martin Pomper \\ Johns Hopkins University School of Medicine \\ Kilian Salerno \\ National Cancer Institute \\ Erica Schott \\ National Cancer Institute \\ Bradford Wood \\ National Cancer Institute \\ Peter Pinto \\ National Cancer Institute \\ Liza Lindenberg \\ National Cancer Institute \\ Peter Choyke \\ National Cancer Institute \\ Deborah Citrin \\ National Cancer Institute \\ Baris Turkbey ( $\nabla$ turkbeyi@mail.nih.gov ) \\ National Cancer Institute, NIH
}

\section{Research}

Keywords: brachytherapy, PSMA, PET, mpMRI, biochemical recurrence 
Posted Date: August 5th, 2020

DOI: https://doi.org/10.21203/rs.3.rs-52861/v1

License: (c) (i) This work is licensed under a Creative Commons Attribution 4.0 International License. Read Full License 


\section{Abstract}

Background and purpose: Both multiparametric magnetic resonance imaging (mpMRI) and prostate specific membrane antigen (PSMA)-targeting positron emission tomography (PET) imaging have shown promise in early localization of prostate cancer $(\mathrm{PCa})$ recurrence after primary external beam radiotherapy. Detecting recurrence after brachytherapy for $\mathrm{PCa}$ using MRI is significantly hampered by susceptibility artifacts secondary to brachy seeds. Here, we compare the efficacy of ${ }^{18}$ F-DCFPyL (2-(3-\{1-carboxy-5-[(6- ${ }^{18}$ F-fluoro-pyridine-3-carbonyl)-amino]-pentyl\}-ureido)-pentanedioic acid) $\mathrm{PET} / \mathrm{CT}$ versus mpMRI for detecting sites of local recurrence after low dose rate (LDR) brachytherapy for prostate cancer.

Materials and methods: A total of 155 patients with a history of recurrent PCa who underwent mpMRI at 3 Tesla and ${ }^{18} \mathrm{~F}-$ DCFPyL PET/CT were retrospectively reviewed. Patients who underwent LDR brachytherapy for PCa and had subsequent biochemical recurrence (BCR) followed by mpMRI and ${ }^{18} \mathrm{~F}$-DCFPyL PET/CT were included in this study. mpMRI was performed on a 3T scanner with endorectal and surface coils and images were prospectively read by a single expert radiologist. The ${ }^{18}$ F-DCFPyL PET/CT scan was prospectively interpreted by one of two nuclear medicine physicians. Patients underwent targeted biopsy when deemed clinically necessary and specimens were interpreted by an expert GU pathologist. Positivity rates (PR) from mpMRI and ${ }^{18} \mathrm{~F}$-DCFPyL PET/CT were compared, and pathology results were used to calculate the positive predictive value (PPV) of each imaging modality for detecting PCa recurrence.

Results: 14 patients who underwent LDR brachytherapy and had subsequent biochemical failure were imaged with mpMRI and ${ }^{18}$ F-DCFPyL PET/CT. 17 lesions were identified on at least one imaging modality. The PR for detection of intraprostatic lesions was 71\% (10/14) for PET/CT and 64.3\% (9/14) for mpMRI. ${ }^{18} \mathrm{~F}$-DCFPyL PET/CT identified pelvic and extra-pelvic lymph nodes in 8 (57\%) patients, while mpMRI noted positive lymph node findings in only 2 (14\%) patients. A total of 14 lesions corresponding to 8 patients were targeted for biopsy and in these, ${ }^{18} \mathrm{~F}$-DCFPyL PET/CT performed better than mpMRI for the detection of local recurrence with a PPV of $81.8 \%$ vs. $66.7 \%$. For the detection of intraprostatic lesions, ${ }^{18} \mathrm{~F}$ DCFPyL PET/CT and mpMRI had a PPV of $87.5 \%$ and $71.4 \%$, respectively.

Conclusion: Our findings suggest that ${ }^{18} \mathrm{~F}-\mathrm{DCFPyL}$ PET/CT detects local and regional recurrent PCa after LDR brachytherapy at a higher rate than mpMRI and has a much higher detection rate of suspicious pelvic lymph nodes. Further studies are needed to validate these findings in larger cohorts.

\section{Background}

Prostate Cancer ( $\mathrm{PCa}$ ) is the most frequently diagnosed cancer in American men, and is the second leading cause of their cancer related deaths with an estimated 174,650 new cases and 31,620 deaths in 2019 (1). There are several established treatment modalities for localized PCa including surgery, external beam radiation therapy [EBRT], brachytherapy, and focal therapies. LDR brachytherapy delivered alone or in combination with EBRT for PCa is selected as initial definitive therapy in up to $40 \%$ of patients with localized prostate cancer (2).

Although efficacy of modern brachytherapy approaches is excellent, biochemical failure occurs in $6 \%$ to $15 \%$ of patients receiving monotherapy with LDR brachytherapy at 10 years (3-7). Early localization of sites of recurrence in these patients is critical to planning further treatment (8). Although patients with biochemical recurrence after definitive radiotherapy were historically managed with temporizing androgen deprivation therapy, numerous potentially curative therapeutic options now exist for patients with isolated local recurrence after radiotherapy, such as focal therapies, reirradiation, HIFU, prostatectomy, and cryotherapy (9-11). These potentially curative salvage therapies carry a substantial risk of treatment related side effects. Thus, accurate localization of recurrence is critical to avoid unnecessary treatment related toxicity of local therapies in patients with regional or distant disease. Additionally, defining patterns of failure after LDR brachytherapy may provide valuable insights into optimal patient selection and seed implantation techniques (12). 
Multiparametric magnetic resonance imaging (mpMRI) consisting of T2-weighted images, apparent diffusion coefficient (ADC) maps, high b-value images, and dynamic contrast enhanced (DCE) imaging, has improved the capacity to identify clinically significant malignant lesions within the prostate (13). mpMRI has been successfully used for the detection of local recurrence following radiotherapy, though it is often avoided in the post-LDR brachytherapy setting due to morphological changes and significant susceptibility artifacts generated by the implanted brachytherapy seeds $(14,15)$. Despite its limitations, few recent studies have demonstrated that mpMRI can still play an important role in the detection of local recurrence after LDR brachytherapy (16).

Recently, novel PET radiotracers targeting prostate specific membrane antigen (PSMA) have shown promise in detecting early biochemical recurrence (17). When combined with CT or MRI, it offers a valuable combination of metabolic and anatomic information for cancer localization. PSMA, a transmembrane protein that is overexpressed in PCa cells but not benign tissue, has been successfully targeted and these PSMA-targeting radiotracers are currently the most sensitive for detection of early recurrence. Several investigational PSMA tracers including PSMA-11 ( $\left.{ }^{68} \mathrm{Ga}-\mathrm{PSMA}\right)$ and ${ }^{18} \mathrm{~F}-\mathrm{DCFPyL}$ have shown to be highly sensitive for detecting sites of local recurrence and metastasis even at low PSA values (18-20).

While mpMRI and ${ }^{18} \mathrm{~F}$-DCFPyL PET/CT have been studied for detecting sites of PCa recurrence in the general prostate cancer population and those who have undergone prostatectomy, there is a lack of published data on the utility of these imaging modalities following treatment with LDR brachytherapy, a setting in which anatomic imaging such as MRI may be compromised due to artifacts $(21,22)$. The objective of this study is to compare the efficacy of mpMRI versus ${ }^{18} \mathrm{~F}-\mathrm{DCFPyL}$ $\mathrm{PET} / \mathrm{CT}$ in the localization of prostate cancer recurrence in patients with biochemical failure after LDR brachytherapy.

\section{Methods}

\section{Patient selection:}

Patients with a history of recurrent PCa who underwent ${ }^{18}$ F-DCFPyL PET/CT and mpMRI between 2015-2019 were retrospectively reviewed. Inclusion criteria were as follows: a) history of PCa and treatment with LDR brachytherapy, b) followed by biochemical recurrence (BCR) (according to the Phoenix criteria (23)), and c) imaging with both mpMRI of the prostate and ${ }^{18}$ F-DCFPyL PET/CT.

\section{Prostate mpMRI and ${ }^{18}$ F-DCFPyL PET/CT imaging protocols:}

mpMRI images were acquired at 3-Tesla (Achieva 3.0T-TX, Philips Healthcare, Best, Netherlands) using endorectal coil (BPX-30, Medrad, Pittsburgh, PA) filled with 45-mL of perfluorocarbon-based fluid (Fomblin, Solvay Specialty Polymers, Milan, Italy) and the anterior half of a 32-channel cardiac SENSE coil (InVivo, Gainesville, FL). The mpMRI sequences obtained included T2W MRI (axial, coronal, and sagittal planes), DWI (ADC map calculated from five evenly spaced bvalues between 0-750 s/mm2 and an acquired high b-value image of b-2000 s/mm2), and DCE MRI. All mpMRI images were prospectively evaluated by a single expert genitourinary radiologist with 13 years of experience in prostate imaging.

${ }^{18} \mathrm{~F}$-DCFPyL PET/CT scans were obtained on a GE Discovery MI DR camera with a $20 \mathrm{~cm}$ coronal and $70 \mathrm{~cm}$ axial field of view. Data were reconstructed with a 3-dimensional Iterative MLEM algorithm using 29 subsets, 3 iterations, time of flight (TOF) mode, PSF regularization parameter 6.0, Gaussian post-filter with $4.1 \mathrm{~cm}$ kernel. Each patient received an IV bolus injection of ${ }^{18}$ F-DCFPyL, mean injected activity of $299.3 \mathrm{MBq}$ [8.09 mCi] (range 229.4-325.6 MBq [6.2-8.8 mCi]), followed by a whole-body PET/CT at 2 hours post-injection (3min/bed position). For attenuation correction and co-registration, lowdose CT scans were obtained (120 kV, $60 \mathrm{mAs}$ ). Standardized uptake values (SUVs) were calculated as the ratio of measured activity to injected dose per kilogram of patient's body weight. PET/CT images were interpreted by one of two board-certified nuclear medicine physicians with over 14 years of experience interpreting PET/CT scans. 


\section{Biopsy technique and pathology analysis:}

Biopsy for pathologic confirmation of PCa recurrence was performed in 8 of 14 patients using transrectal ultrasound (TRUS)-guided 12 core systematic biopsy and/or MRI/TRUS fusion guided biopsy. Biopsies were performed by one of two physicians (one urologist and one interventional radiologist), each with over ten years of experience in systematic and fusion guided prostate biopsy. Biopsy specimens were evaluated by a single expert genitourinary pathologist.

\section{Statistical analysis:}

Positivity rates (PR) (24) were calculated for each imaging modality for various anatomic locations by dividing the number of patients with positive findings in each anatomical area [intraprostatic, seminal vesicle (SV), penile bulb, bladder wall, pelvic lymph nodes (LN), extra-pelvic LN] by the total number of patients imaged with that modality. Lesions with available pathology results (ground truth) were used to calculate the positive predictive values (PPV) for both mpMRI and ${ }^{18} \mathrm{~F}$ DCFPyL PET/CT. PPV were calculated for each imaging modality for detection of local recurrence within the prostate gland and within the local region of the pelvis overall.

\section{Results}

A total of 14 patients with history of PCa who were treated with brachytherapy alone $(n=6)$ or with supplemental external beam radiation therapy (EBRT) $(n=8)$ were imaged with both mpMRI and ${ }^{18} \mathrm{~F}$-DCFPyL PET/CT secondary to BCR according to Phoenix criteria. Patient demographics are summarized in Table 1. The median age of the cohort was 68 years (range 56-78), and the median time to BCR following brachytherapy was 4.66 years (range 0.9-13.2). The median (range) PSA values prior to treatment and at the time of imaging were $7.50 \mathrm{ng} / \mathrm{ml}(4.2-49.5)$ and $4.32 \mathrm{ng} / \mathrm{ml}(2.13-28.9)$, respectively. Gleason grade group prior to brachytherapy ranged from $1-5$ with a majority of patients $(93 \%, 13 / 14)$ having Gleason grade group 1-3 cancer at baseline.

All patients had at least one positive finding on one or both imaging modalities (Fig. 1-3). Table 2 summarizes individual patient clinical characteristics, sites of recurrence, imaging findings, and associated biopsy results when available. 8 patients (57\%) underwent biopsy of suspicious lesions, resulting in corresponding pathology results for 14 local pelvic lesions (9 prostate lesions, 2 SV lesions, 1 penile bulb lesion, 1 bladder wall lesion, and 1 pelvic lymph node).

A total of 11 intraprostatic lesions were identified on imaging in 10 patients. The positivity rate for intraprostatic lesions was $71.4 \%$ (10/14) for ${ }^{18}$ F-DCFPyL PET/CT and 64.3\% (9/14) for mpMRI. Eight of the 11 identified intraprostatic lesions were positive on both mpMRI and PET/CT, 1 was positive on mpMRI only (pathology proven false positive), and 2 were positive on PET/CT only (pathology proven true positive). In the group that did not receive EBRT, $5 / 6$ patients (83\%) had an intraprostatic lesion identified and all lesions were positive on both imaging modalities. Of the 8 patients who received EBRT, the PR for detecting intraprostatic lesions was $50 \%$ for both PET/CT and mpMRI. Table 3 summarizes the positivity rates of each imaging modality for intraprostatic lesions, SV lesions, and other positive findings.

The PR for SV lesions was also similar between the two imaging modalities, with a PR of 22\% (3/14) on PET/CT and $14 \%$ (2/14) on mpMRI. 2 SV lesions were positive on both mpMRI and PET/CT, and 1 SV lesion was positive on PET/CT alone. Associated pathology results (available for 2 out of 3 of the SV lesions) revealed cancer in both the MRI(-)/PET(+) SV lesion and the $\mathrm{MRI}(+) / \mathrm{PET}(+)$ lesion. (Table 4)

${ }^{18} \mathrm{~F}$-DCFPyL identified a colonic lesion and a penile bulb lesion in separate patients which were both negative on mpMRI. The colonic lesion was located in the ascending colon; however, it was not biopsied as the patient declined. The penile bulb lesion was biopsied with pathology confirming prostatic adenocarcinoma. ${ }^{18} \mathrm{~F}$-DCFPyL also detected pelvic and extrapelvic lymph nodes at a higher rate than mpMRI. The PR for mpMRI for detection of pelvic lymph nodes was $14.3 \%(2 / 14)$ 
and no extra-pelvic LNs were reported. In contrast, the PRs for pelvic and extra-pelvic LN detection with ${ }^{18} \mathrm{~F}$-DCFPyL PET/CT were 57.1\% (8/14) and 42.9\% (6/14), respectively. The majority of LN identified were multiple, deep, and located near vasculature, preventing safe biopsy. A single LN biopsy was performed for an MRI(-)/PET(+) pelvic node, and pathologic analysis revealed benign inflammation.

Biopsy results from 8 patients and 14 sampled lesions were used to calculate the PPV of each imaging modality for detection of local recurrence. The PPV of mpMRI for detecting intraprostatic recurrence was $71.43 \%$, compared to $87.50 \%$ for ${ }^{18}$ F-DCFPyL PET/CT. Of the 9 intraprostatic lesions that were biopsied, 8 had accurate matching PET/CT findings (7 true positives, 1 true negative, 0 false negative, and 1 false positive). The false positive lesion revealed inflammation on biopsy and the same lesion was also reported as positive on mpMRI. This level of diagnostic accuracy contrasts with the mpMRI findings for the sampled intraprostatic lesions, which included 5 true positives, 0 true negatives, 2 false negatives, and 2 false positives. The PPV of mpMRI for detecting overall local pelvic recurrence is $66.67 \%$ compared to $81.82 \%$ for ${ }^{18} \mathrm{~F}$-DCFPyL PET/CT. All calculated PPVs are summarized in Table 5.

Table 1

Baseline demographics of the study population.

\begin{tabular}{|ll|}
\hline Parameter & Median (Range) / n (\%) \\
\hline Age (years) & $68(56-78)$ \\
\hline Pre-treatment PSA $(\mathrm{ng} / \mathrm{ml})$ & $4.66(0.9-13.2)$ \\
\hline Initial Gleason Grade Group & \\
\hline 1 & $6(43 \%)$ \\
\hline 2 & $4(29 \%)$ \\
\hline 3 & $3(21 \%)$ \\
\hline 4 & $0(0 \%)$ \\
\hline 5 & $1(7 \%)$ \\
\hline Time to BCR (years) & $4.66(0.9-13.2)$ \\
\hline PSA at imaging $(\mathrm{ng} / \mathrm{ml})$ & $4.32(2.13-28.9)$ \\
\hline
\end{tabular}


Table 2

Individual patient characteristics, sites of recurrence, imaging findings, and associated biopsy results (if available).

\begin{tabular}{|c|c|c|c|c|c|c|c|c|c|c|}
\hline $\begin{array}{l}\text { Patient } \\
\#\end{array}$ & $\begin{array}{l}\text { Age } \\
\text { (years) }\end{array}$ & $\begin{array}{l}\text { Pre- } \\
\text { BT } \\
\text { GGG }\end{array}$ & $\begin{array}{l}\text { Pre-BT } \\
\text { PSA } \\
(\mathrm{ng} / \mathrm{ml})\end{array}$ & $\begin{array}{l}\text { PSA at } \\
\text { mpMRI } \\
(\mathrm{ng} / \mathrm{ml})\end{array}$ & $\begin{array}{l}\text { Days } \\
\text { between } \\
\text { mpMRI } \\
\text { and } \\
\text { PET/CT }\end{array}$ & EBRT? & $\begin{array}{l}\text { Lesion } \\
\text { Location }\end{array}$ & mpMRI & PET/CT & Pathology \\
\hline \multirow[t]{5}{*}{1} & 72 & 3 & 49.46 & 28.85 & 3 & yes & prostate & + & + & NA \\
\hline & & & & & & & SV & + & + & NA \\
\hline & & & & & & & pelvic LN & - & + & NA \\
\hline & & & & & & & $\begin{array}{l}\text { extrapelvic } \\
\text { LN }\end{array}$ & - & + & NA \\
\hline & & & & & & & colon & - & + & NA \\
\hline 2 & 76 & 1 & 4.2 & 2.69 & 4 & no & prostate & + & + & NA \\
\hline 3 & 60 & 1 & 4.5 & 11.85 & 7 & yes & prostate & + & + & + \\
\hline \multirow[t]{3}{*}{4} & 57 & 2 & 6.9 & 9.12 & 366 & no & prostate & + & + & + \\
\hline & & & & & & & pelvic LN & - & + & NA \\
\hline & & & & & & & $\begin{array}{l}\text { extrapelvic } \\
\text { LN }\end{array}$ & - & + & NA \\
\hline 5 & 69 & 1 & 5.4 & 3 & 2 & no & prostate & + & + & + \\
\hline \multirow[t]{3}{*}{6} & 70 & 2 & 7.5 & 6.13 & 35 & yes & prostate & - & + & + \\
\hline & & & & & & & prostate & - & + & + \\
\hline & & & & & & & SV & - & + & + \\
\hline 7 & 77 & 3 & 14.14 & 3.85 & 2 & yes & prostate & + & + & + \\
\hline \multirow[t]{3}{*}{8} & 68 & 1 & 7.9 & 6.71 & 18 & no & prostate & + & + & + \\
\hline & & & & & & & $\begin{array}{l}\text { penile } \\
\text { bulb }\end{array}$ & - & + & + \\
\hline & & & & & & & $\begin{array}{l}\text { bladder } \\
\text { wall }\end{array}$ & - & - & + \\
\hline \multirow[t]{2}{*}{9} & 78 & 1 & NA & 3.26 & 1 & yes & pelvic LN & - & + & NA \\
\hline & & & & & & & $\begin{array}{l}\text { extrapelvic } \\
\text { LN }\end{array}$ & - & + & NA \\
\hline \multirow[t]{2}{*}{10} & 56 & 2 & 8.4 & NA & 146 & no & pelvic LN & + & + & NA \\
\hline & & & & & & & $\begin{array}{l}\text { extrapelvic } \\
\text { LN }\end{array}$ & - & + & NA \\
\hline 11 & 56 & 1 & 6.6 & 4.32 & 44 & yes & pelvic LN & + & + & NA \\
\hline \multirow[t]{2}{*}{12} & 67 & 3 & 8.4 & 2.13 & 25 & yes & pelvic LN & - & + & NA \\
\hline & & & & & & & $\begin{array}{l}\text { extrapelvic } \\
\text { LN }\end{array}$ & - & + & NA \\
\hline
\end{tabular}




\begin{tabular}{|c|c|c|c|c|c|c|c|c|c|c|}
\hline $\begin{array}{l}\text { Patient } \\
\#\end{array}$ & $\begin{array}{l}\text { Age } \\
\text { (years) }\end{array}$ & $\begin{array}{l}\text { Pre- } \\
\text { BT } \\
\text { GGG }\end{array}$ & $\begin{array}{l}\text { Pre-BT } \\
\text { PSA } \\
\text { (ng/ml) }\end{array}$ & $\begin{array}{l}\text { PSA at } \\
\text { mpMRI } \\
(n g / m l)\end{array}$ & $\begin{array}{l}\text { Days } \\
\text { between } \\
\text { mpMRI } \\
\text { and } \\
\text { PET/CT }\end{array}$ & EBRT? & $\begin{array}{l}\text { Lesion } \\
\text { Location }\end{array}$ & mpMRI & $\mathrm{PET} / \mathrm{CT}$ & Pathology \\
\hline \multirow[t]{4}{*}{13} & 65 & 5 & 5.6 & 2.86 & 14 & yes & prostate & + & - & - \\
\hline & & & & & & & SV & + & + & + \\
\hline & & & & & & & pelvic LN & - & + & NA \\
\hline & & & & & & & $\begin{array}{l}\text { extrapelvic } \\
\text { LN }\end{array}$ & - & + & NA \\
\hline \multirow[t]{2}{*}{14} & 73 & 2 & 10.85 & 10.27 & 28 & no & prostate & + & + & - \\
\hline & & & & & & & pelvic LN & - & + & - \\
\hline
\end{tabular}

Table 3

Positivity rates for mpMRI and DCFPyL PET/CT for detection of lesions in various anatomic locations $(N=14)$.

\begin{tabular}{|lll|}
\hline Anatomic Location & mpMRI PR & PET/CT PR \\
\hline Non-Lymph Node Findings & $\mathrm{n}(\%)$ & $\mathrm{n}(\%)$ \\
\hline Prostate & $9(64.3 \%)$ & $10(71.4 \%)$ \\
\hline Seminal Vesicle & $2(14.3 \%)$ & $3(21.4 \%)$ \\
\hline Colon & $0(0 \%)$ & $1(7.1 \%)$ \\
\hline Penile Bulb & $0(0 \%)$ & $1(7.1 \%)$ \\
\hline Lymph Node Findings & & \\
\hline Pelvic Lymph Node(s) & $2(14.3 \%)$ & $8(57.1 \%)$ \\
\hline Extra-Pelvic Lymph Node(s) & $0(0 \%)$ & $6(42.9 \%)$ \\
\hline
\end{tabular}


Table 4

Identified lesions stratified by positivity on each imaging modality for each anatomic location.

\begin{tabular}{|lllll|}
\hline Lesion Location & Total Identified & MRI +/PET + & MRI +/PET - & MRI -/PET + \\
\hline Non-Lymph Node Findings & $\mathrm{N}$ & $\mathrm{n}(\%)$ & $\mathrm{n}(\%)$ & $\mathrm{n}(\%)$ \\
\hline Prostate & 11 & $8(73 \%)$ & $1(9 \%)$ & $2(18 \%)$ \\
\hline Seminal Vesicle & 3 & $2(67 \%)$ & $0(0 \%)$ & $1(33 \%)$ \\
\hline Colon & 1 & $0(0 \%)$ & $0(0 \%)$ & $1(100 \%)$ \\
\hline Penile Bulb & 1 & $0(0 \%)$ & $0(0 \%)$ & $1(100 \%)$ \\
\hline Lymph Node Findings, patient level & & & & \\
\hline Pelvic Lymph Node(s) & 8 & $2(25 \%)$ & $0(0 \%)$ & $6(75 \%)$ \\
\hline Extra-Pelvic Lymph Node(s) & 6 & $0(0 \%)$ & $0(0 \%)$ & $6(100 \%)$ \\
\hline
\end{tabular}

Table 5

Positive predictive values for each

imaging modality for recurrent

cancer detection after brachytherapy.

*"All local pelvic lesions" includes

lesions within the prostate, seminal

vesicles, penile bulb, and pelvic

lymph nodes.

\begin{tabular}{|ll|}
\hline Imaging Modality & PPV \\
\hline Intraprostatic lesions & \\
\hline mpMRI & $71.43 \%$ \\
\hline DCFPyL PET-CT & $87.50 \%$ \\
\hline All local pelvic lesions* & \\
\hline mPMRI & $66.67 \%$ \\
\hline DCFPyL PET-CT & $81.82 \%$ \\
\hline
\end{tabular}

\section{Discussion}

We compared the efficacy of mpMRI and ${ }^{18} \mathrm{~F}-\mathrm{DCFPyL}$ PET/CT for the detection of recurrent PCa lesions in patients who were treated with LDR brachytherapy and subsequently experienced biochemical failure. In our study, overall positivity rates were similar among the two modalities for intraprostatic and seminal vesicle lesions, however, biopsy and histopathology analysis revealed a higher PPV for ${ }^{18} \mathrm{~F}$-DCFPyL PET/CT. Additionally, ${ }^{18} \mathrm{~F}$-DCFPyL PET/CT had a higher positivity rate for the detection of pelvic and extra-pelvic lymph nodes than mpMRI, which can be attributed to a greater dependence on size criteria $(>1 \mathrm{~cm})$ in evaluation of lymph nodes by $\mathrm{mpMRI}$ and the limited coverage of MRI compared to PET/CT.

There are limited studies in the literature evaluating or comparing these two imaging modalities in patients with recurrence after LDR brachytherapy. Unlike other treatment modalities, LDR brachytherapy results in a substantial degree of artifact 
from the numerous permanently implanted brachytherapy seeds within the prostate. Although these seeds can result in mild CT scatter, they substantially impact the quality of MRI. In several studies, PET/CT was found to perform better than mpMRI for the identification extraprostatic disease. In one recent study, Liu et al demonstrated that ${ }^{18}$ F-DCFPyL PET revealed extraprostatic cancer foci in twice as many men with radio-recurrent prostate cancer compared with conventional imaging, which included pelvic MRIs. ${ }^{18}$ F-DCFPyL PET detected intraprostatic recurrence in $87 \%$ of men compared with $67 \%$ with MRI. However, this study included multiple types of radiation therapy including EBRT and brachytherapy; moreover there was no histopathologic validation for the intraprostatic foci suspicious for recurrence (25).

mpMRI has played a large and increasing role in localized prostate cancer management over the past couple of decades due to its ability to provide exceptional anatomic detail. However, in patients who have undergone LDR brachytherapy, the diagnosis of local recurrence on mpMRI is challenging secondary to the susceptibility artifacts related to implanted seeds and therapeutic glandular changes (i.e. decrease in glandular size and diffusely heterogeneous prostatic signal intensity pattern in MRI) $(15,26)$. This is reflected in the relatively poor performance of mpMRI in this study which demonstrated an overall PPV of only $67 \%$. While the PR for intraprostatic lesions was high (71.43\%), biopsy results revealed cancer in 2 lesions which were $\mathrm{MRI}(-)$ and benign tissue in 2 out of $9 \mathrm{mpMRI}(+)$ lesions which lowered the PPV.

In contrast, $\mathrm{PET} / \mathrm{CT}$ is a molecular imaging study which relies less on anatomic features and more on cellular biology and is thus less impacted by seed implantation. For intraprostatic lesions, ${ }^{18} \mathrm{~F}$-DCFPyL PET/CT had a PPV of $87.5 \%$. This reflects the lower rate of false positives associated with PET/CT compared to mpMRI. The finding of one false negative lesion on ${ }^{18} \mathrm{~F}$-DCFPyL PET/CT, a bladder wall lesion, speaks to the high sensitivity of this imaging technique. Lesions near the bladder wall may be more challenging to detect given the urinary excretion of the radiotracer.

Multiple studies have confirmed a high detection rate of recurrent PCa with various PSMA-targeting radiotracers even in biochemical recurrence patients with low PSA levels, and usually exceeds mpMRI (27-29). Some studies suggest a strong correlation between PSA levels and diagnostic performance of PSMA-targeting PET tracers (27-31). Our small sample size cohort did not allow for such a correlation, however in a future study with a larger cohort, imaging results should be correlated with PSA levels to test whether this holds true in patients who have undergone brachytherapy.

Previous studies have commented on the benefit of combined use of mpMRI and PSMA PET/CT for more accurate diagnosis of foci of local recurrence after radical prostatectomy or primary radiotherapy $(12,32,33)$. In our cohort, we observed a similar benefit. While MRI was able to deliver high resolution anatomic scans, PSMA PET/CT illustrated cancer specific uptake. This combination resulted in a radiologic diagnosis of recurrence in most of our patients. An important advantage of PSMA PET/CT was the higher positivity rate to identify lymph node involvement. The finding of disease in extraprostatic sites changes the potential treatments appropriate for the patient.

Biochemical recurrence after radiation therapy has been traditionally managed by use of conventional staging and imaging methods such as computed tomography and bone scan with subsequent initiation of androgen deprivation therapy (34). Use of more novel and dedicated imaging techniques such as mpMRI combined with PSMA PET/CT has been documented to better depict and map the disease burden in prostate cancer patients with BCR (35). This robust imaging approach can also aid delivering personalized salvage treatment instead of standalone systematic androgen deprivation. Few groups have reported promising results on utility of using PSMA PET/CT guided salvage radiation therapy for patients with BCR $(36,37)$. Although we did not perform a formal analysis, several of the patients included in our study were found to have isolated local recurrence and were found to be eligible to enroll on trials of salvage local therapy.

There are several limitations to this study. First, thorough statistical methods were impossible with the small study population, but relevant clinical analysis was performed. Secondly, there was a lack of uniformity in the treatment history of our cohort, with 8/14 patients receiving both brachytherapy and EBRT. Additionally, not all patients had corresponding biopsy results, and lymph nodes were not biopsied uniformly. Lymph node biopsy can often be challenging in this setting

Page 10/16 
given the proximity to critical structures, lack of enlargement of the suspected lymph node, and the confines of the bony pelvis. Additionally, biopsy was not performed if the results would not affect disease management. The findings could also be confounded by the referral bias. Salvage systemic treatment is usually commenced early in patients with rapid PSA doubling time after onset of biochemical failure as these patients have a higher predilection for distant metastasis $(38,39)$. No patient in this study was on ADT at the time of imaging, therefore, it is likely that the majority of our patients had relatively slower PSA kinetics which could affect the preponderance of local failure in our study. Finally, all imaging data were interpreted by experts with significant experience in mpMRI and PSMA PET which could limit the reproducibility of results in other centers.

\section{Conclusion}

In conclusion, we demonstrate that ${ }^{18} \mathrm{~F}$-DCFPyL PET/CT is superior to mpMRI for identifying sites of recurrence in patients with suspected PCa recurrence after LDR brachytherapy with or without external beam radiotherapy, with higher PPV and greater detection of suspicious lymph nodes. Further studies with large patient population and more robust histopathological sampling are necessary to validate our findings.

\section{Declarations}

\section{Ethics approval and consent to participate:}

All procedures performed in studies involving human participants were in accordance with the ethical standards of the institutional and/or national research committee and with the 1964 Helsinki declaration and its later amendments or comparable ethical standards.

Informed consent was obtained from all individual participants included in the study.

\section{Consent for publication:}

All co-authors confirm that they agree with publication of this manuscript.

\section{Availability of data and material:}

The datasets used and/or analyzed during the current study are available from the corresponding author on reasonable request.

\section{Funding:}

This research is funded by intramural research program of $\mathrm{NIH}$,

\section{Conflict of Interest:}

Author BJW is supported by the Intramural Research Program of the $\mathrm{NIH}$ and the $\mathrm{NIH}$ Center for Interventional Oncology and NIH Grant \# Z1A CL040015-08. NIH and Philips/InVivo Inc have a cooperative Research and Development Agreement. $\mathrm{NIH}$ and Philips / InVivo Inc have a patent license agreement and NIH and BJW, BT, PAP, PLC may receive royalties. MGP, and RCM, are co-inventors on a US Patent covering [18F]DCFPyL and as such are entitled to a portion of any licensing fees 
and royalties generated by this technology. This arrangement has been reviewed and approved by the Johns Hopkins University in accordance with its conflict of interest policies. The remaining authors have no disclosures.

\section{Acknowledgements:}

N/A.

\section{Authors' contributions:}

Conception and design: SMW, PLC, DC, BT

Collection and assembly of data: SMW, MF, SR, JS, ES, LL, DC, BT

Data analysis and interpretation: SMW, MF, SR, JS, ES, KS, LL, DC, BT

Manuscript writing/editing: All authors

Final approval of manuscript: All authors

\section{References}

1. Siegel RL, Miller KD, Jemal A. Cancer statistics, 2019. CA Cancer J Clin. 2019;69(1):7-34.

2. Marcus DM, Jani AB, Godette K, Rossi PJJJotNMA. A review of low-dose-rate prostate brachytherapy-Techniques and outcomes. 2010;102(6):500-10.

3. Martell K, Husain S, Taussky D, Angyalfi S, Delouya G, Després P, et al. Multicenter Evaluation of Biochemical RelapseFree Survival Outcomes for Intraoperatively Planned Prostate Brachytherapy Using an Automated Delivery System. International Journal of Radiation Oncology Biology Physics. 2017;99:895-903.

4. Morris WJ, Keyes M, Spadinger I, Kwan W, Liu M, McKenzie M, et al. Population-based 10-year oncologic outcomes after low-dose-rate brachytherapy for low-risk and intermediate-risk prostate cancer. Cancer. 2013;119:1537-46.

5. Routman DM, Funk RK, Stish BJ, Mynderse LA, Wilson TM, McLaren R, et al. Permanent prostate brachytherapy monotherapy with I-125 for low- and intermediate-risk prostate cancer: Outcomes in 974 patients. Brachytherapy. 2019;18:1-7.

6. Fellin G, Mirri MA, Santoro L, Jereczek-Fossa BA, Divan C, Mussari S, et al. Low dose rate brachytherapy (LDR-BT) as monotherapy for early stage prostate cancer in Italy: practice and outcome analysis in a series of 2237 patients from 11 institutions. The British journal of radiology. 2016;89(1065):20150981.

7. Lazarev S, Thompson MR, Stone NN, Stock RG. Low-dose-rate brachytherapy for prostate cancer: outcomes at $>10$ years of follow-up. BJU international. 2018;121(5):781-90.

8. Lacy JM, Wilson WA, Bole R, Chen L, Meigooni AS, Rowland RG, et al. Salvage brachytherapy for biochemically recurrent prostate cancer following primary brachytherapy. 2016;2016.

9. Giannarini G, Fossati N, Gandaglia G, Cucchiara V, Ficarra V, Mirone V, et al. Will image-guided metastasis-directed therapy change the treatment paradigm of oligorecurrent prostate cancer? 2018.

10. Walker SM, Lim I, Lindenberg L, Mena E, Choyke PL, Turkbey B. Positron emission tomography (PET) radiotracers for prostate cancer imaging. Abdominal radiology (New York). 2020.

11. Tetreault-Laflamme A, Crook J. Options for Salvage of Radiation Failures for Prostate Cancer. Seminars in radiation oncology. 2017;27(1):67-78. 
12. Valle LF, Greer MD, Shih JH, Barrett T, Law YM, Rosenkrantz AB, et al. Multiparametric MRI for the detection of local recurrence of prostate cancer in the setting of biochemical recurrence after low dose rate brachytherapy. Diagn Interv Radiol. 2018;24(1):46-53.

13. Damber J-E, Aus G. Prostate cancer. Lancet. 2008;371(9625):1710-21.

14. Barchetti F, Panebianco V. Multiparametric MRI for recurrent prostate cancer post radical prostatectomy and postradiation therapy. Biomed Res Int. 2014;2014:316272-.

15. Gaur S, Turkbey B. Prostate MR Imaging for Posttreatment Evaluation and Recurrence. Urol Clin North Am. 2018;45(3):467-79.

16. Valle LF, Greer MD, Shih JH, Barrett T, Law YM, Rosenkrantz AB, et al. Multiparametric MRI for the detection of local recurrence of prostate cancer in the setting of biochemical recurrence after low dose rate brachytherapy. Diagnostic and interventional radiology (Ankara, Turkey). 2018;24(1):46-53.

17. Tan N, Bavadian N, Calais J, Oyoyo U, Kim J, Turkbey IB, et al. Imaging of Prostate Specific Membrane Antigen Targeted Radiotracers for the Detection of Prostate Cancer Biochemical Recurrence after Definitive Therapy: A Systematic Review and Meta-Analysis. The Journal of urology. 2019;202(2):231-40.

18. Rousseau E, Wilson D, Lacroix-Poisson F, Krauze A, Chi K, Gleave M, et al. A Prospective Study on (18)F-DCFPyL PSMA $\mathrm{PET} / \mathrm{CT}$ Imaging in Biochemical Recurrence of Prostate Cancer. Journal of nuclear medicine : official publication, Society of Nuclear Medicine. 2019;60(11):1587-93.

19. Rowe SP, Macura KJ, Mena E, Blackford AL, Nadal R, Antonarakis ES, et al. PSMA-based [18 F] DCFPyL PET/CT is superior to conventional imaging for lesion detection in patients with metastatic prostate cancer. 2016;18(3):411-9.

20. Koerber SA, Will L, Kratochwil C, Haefner MF, Rathke H, Kremer C, et al. 68Ga-PSMA-11 PET/CT in Primary and Recurrent Prostate Carcinoma: Implications for Radiotherapeutic Management in 121 Patients. 2019;60(2):234-40.

21. Mena E, Lindenberg ML, Turkbey IB, Shih JH, Harmon SA, Lim I, et al. 18F-DCFPyL PET/CT Imaging in Patients with Biochemical Recurrence Prostate Cancer after Primary Local Therapy. 2019.

22. Rowe SP, Campbell SP, Mana-Ay M, Szabo Z, Allaf ME, Pienta KJ, et al. Prospective Evaluation of PSMA-Targeted 18FDCFPyL PET/CT in Men with Biochemical Failure after Radical Prostatectomy for Prostate Cancer. 2019.

23. Roach III M, Hanks G, Thames Jr H, Schellhammer P, Shipley WU, Sokol GH, et al. Defining biochemical failure following radiotherapy with or without hormonal therapy in men with clinically localized prostate cancer: recommendations of the RTOG-ASTRO Phoenix Consensus Conference. 2006;65(4):965-74.

24. Savir-Baruch B, Lovrec P, Solanki AA, Adams WH, Yonover PM, Gupta G, et al. Fluorine-18-Labeled Fluciclovine PET/CT in Clinical Practice: Factors Affecting the Rate of Detection of Recurrent Prostate Cancer. American Journal of Roentgenology. 2019:1-8.

25. Liu W, Zukotynski K, Emmett L, Chung HT, Chung P, Wolfson R, et al. A Prospective Study of 18F-DCFPyL PSMA $\mathrm{PET} / \mathrm{CT}$ Restaging in Recurrent Prostate Cancer following Primary External Beam Radiotherapy or Brachytherapy. International journal of radiation oncology, biology, physics. 2020;106(3):546-55.

26. Lee SL, Ravi A, Morton G, Loblaw A, Tseng CL, Haider M, et al. Changes in ADC and T2-weighted MRI-derived radiomic features in patients treated with focal salvage HDR prostate brachytherapy for local recurrence after previous externalbeam radiotherapy. Brachytherapy. 2019;18(5):567-73.

27. Fendler WP, Calais J, Eiber M, Flavell RR, Mishoe A, Feng FY, et al. Assessment of 68Ga-PSMA-11 PET Accuracy in Localizing Recurrent Prostate Cancer: A Prospective Single-Arm Clinical Trial. JAMA Oncol. 2019;5(6):856-63.

28. Hoffmann MA, Buchholz H-G, Wieler HJ, Höfner T, Müller-Hübenthal J, Trampert L, et al. The positivity rate of 68Gallium-PSMA-11 ligand PET/CT depends on the serum PSA-value in patients with biochemical recurrence of prostate cancer. Oncotarget. 2019;10(58):6124-37.

29. Mena E, Lindenberg ML, Turkbey IB, Shih JH, Harmon SA, Lim I, et al. (18)F-DCFPyL PET/CT Imaging in Patients with Biochemical Recurrence Prostate Cancer after Primary Local Therapy. J Nucl Med. 2019:jnumed.119.234799.

Page $13 / 16$ 
30. Farolfi A, Gafita A, Calais J, Eiber M, Afshar-Oromieh A, Spohn F, et al. (68)Ga-PSMA-11 Positron Emission Tomography Detects Residual Prostate Cancer after Prostatectomy in a Multicenter Retrospective Study. The Journal of urology. 2019;202(6):1174-81.

31. Song H, Harrison C, Duan H, Guja K, Hatami N, Franc B, et al. Prospective Evaluation in an Academic Center of (18)FDCFPyL PET/CT in Biochemically Recurrent Prostate Cancer: A Focus on Localizing Disease and Changes in Management. Journal of nuclear medicine : official publication, Society of Nuclear Medicine. 2019:jnumed.119.231654.

32. Muller BG, Kaushal A, Sankineni S, Lita E, Hoang AN, George AK, et al. Multiparametric magnetic resonance imagingtransrectal ultrasound fusion-assisted biopsy for the diagnosis of local recurrence after radical prostatectomy. Urol Oncol. 2015;33(10):425.e1-.e6.

33. Mertan FV, Greer MD, Borofsky S, Kabakus IM, Merino MJ, Wood BJ, et al. Multiparametric Magnetic Resonance Imaging of Recurrent Prostate Cancer. Top Magn Reson Imaging. 2016;25(3):139-47.

34. Crook JM, O'Callaghan CJ, Duncan G, Dearnaley DP, Higano CS, Horwitz EM, et al. Intermittent androgen suppression for rising PSA level after radiotherapy. The New England journal of medicine. 2012;367(10):895-903.

35. Lindenberg L, Mena E, Turkbey B, Shih JH, Reese SE, Harmon SA, et al. Evaluating Biochemically Recurrent Prostate Cancer: Histologic Validation of (18)F-DCFPyL PET/CT with Comparison to Multiparametric MRI. Radiology. 2020:192018.

36. Artigas C, Flamen P, Charlier F, Levillain H, Wimana Z, Diamand R, et al. (68)Ga-PSMA PET/CT-based metastasisdirected radiotherapy for oligometastatic prostate cancer recurrence after radical prostatectomy. World journal of urology. 2019;37(8):1535-42.

37. Henkenberens C, von Klot CA, Ross TL, Bengel FM, Wester HJ, Merseburger AS, et al. (68)Ga-PSMA ligand PET/CTbased radiotherapy in locally recurrent and recurrent oligometastatic prostate cancer : Early efficacy after primary therapy. Strahlentherapie und Onkologie : Organ der Deutschen Rontgengesellschaft [et al]. 2016;192(7):431-9.

38. Antonarakis ES, Chen Y, Elsamanoudi SI, Brassell SA, Da Rocha MV, Eisenberger MA, et al. Long-term overall survival and metastasis-free survival for men with prostate-specific antigen-recurrent prostate cancer after prostatectomy: analysis of the Center for Prostate Disease Research National Database. BJU international. 2011;108(3):378-85.

39. Lee AK, Levy LB, Cheung R, Kuban D. Prostate-specific antigen doubling time predicts clinical outcome and survival in prostate cancer patients treated with combined radiation and hormone therapy. Int J Radiat Oncol Biol Phys. 2005;63(2):456-62.

\section{Figures}



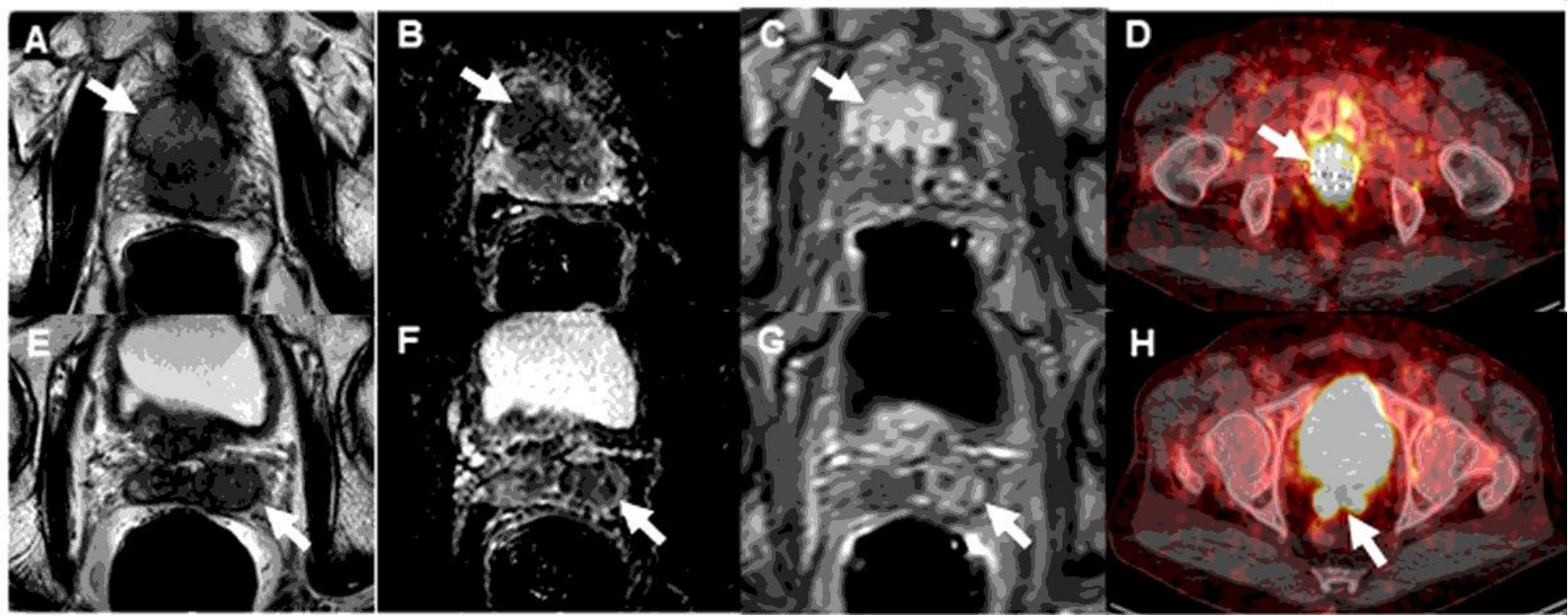

\section{Figure 1}

72-year-old patient with history of biochemical recurrence of prostate cancer 4.75 years following brachytherapy; PSA at time of imaging was $28.85 \mathrm{ng} / \mathrm{mL}$. mpMRI reveals a large hypointense lesion in the right mid-base anterior transition zone (A) which is markedly hypointense on ADC map (B) and has associated early focal enhancement on DCE MRI (C). The prostate demonstrates increased uptake on 18F-DCFPyL PET/CT (D) (arrows). Additionally, the seminal vesicles are seen invaded on T2W MRI (E), with associated positive findings on ADC map (F), DCE MRI (G), and 18F-DCFPyL (H) (arrows). Targeted biopsy of the prostate lesion and seminal vesicles demonstrated recurrent adenocarcinoma.

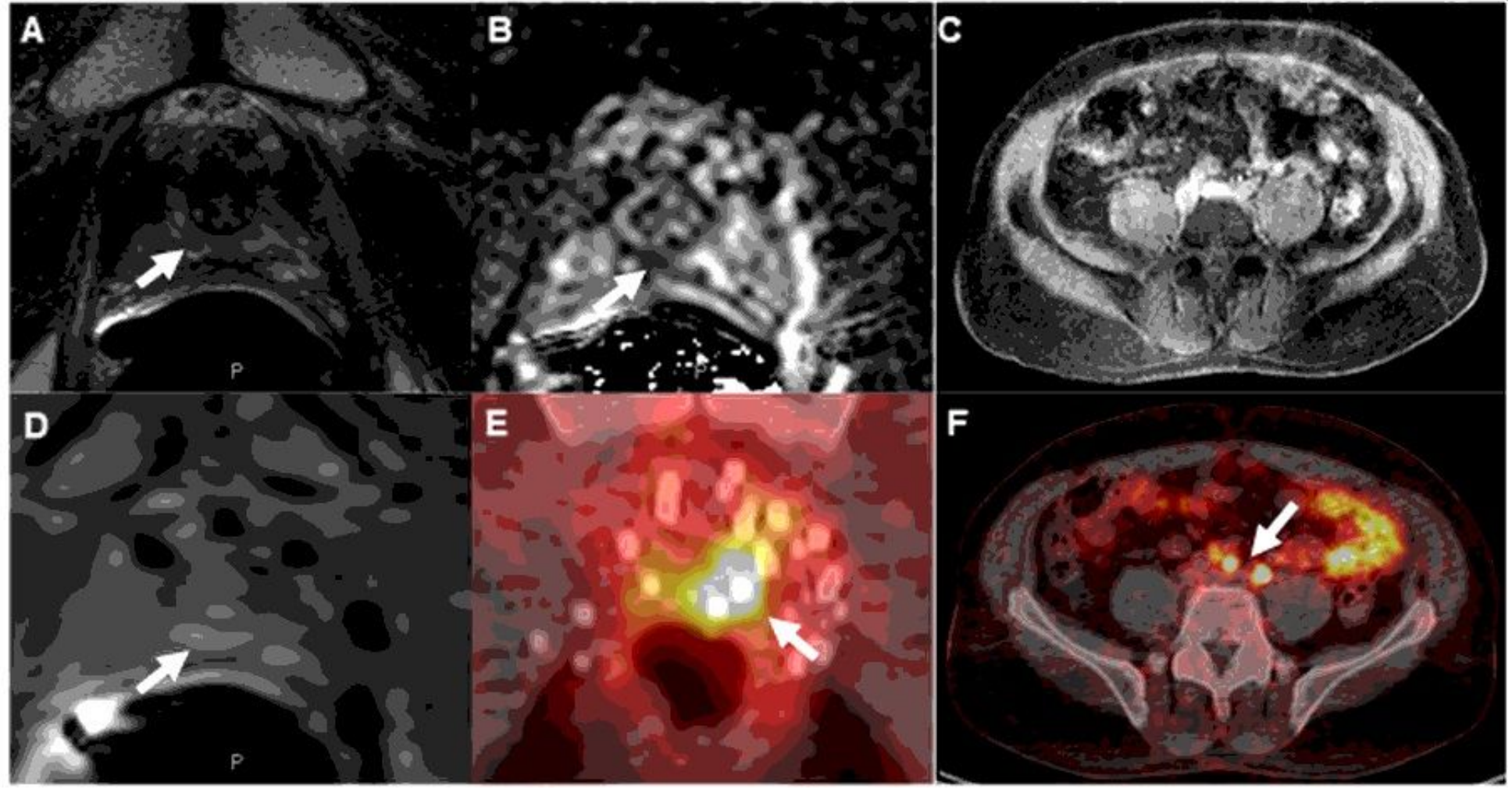

Figure 2

57-year-old patient with history of biochemical recurrence of prostate cancer 3.9 years following brachytherapy; PSA at time of imaging was $9.12 \mathrm{ng} / \mathrm{mL}$. mpMRI reveals a hypointense lesion in the midline distal apical peripheral zone (A) which 
is more readily seen on ADC map (B) and has associated early focal enhancement on DCE imaging (D) (arrows). The prostate lesion demonstrates increased uptake on 18F-DCFPyL PET/CT (E) (arrow). Additionally, two left common iliac nodes are seen on DCFPyL PET/CT with avid uptake (F) (arrow) which were not prospectively identified on MRI (C).

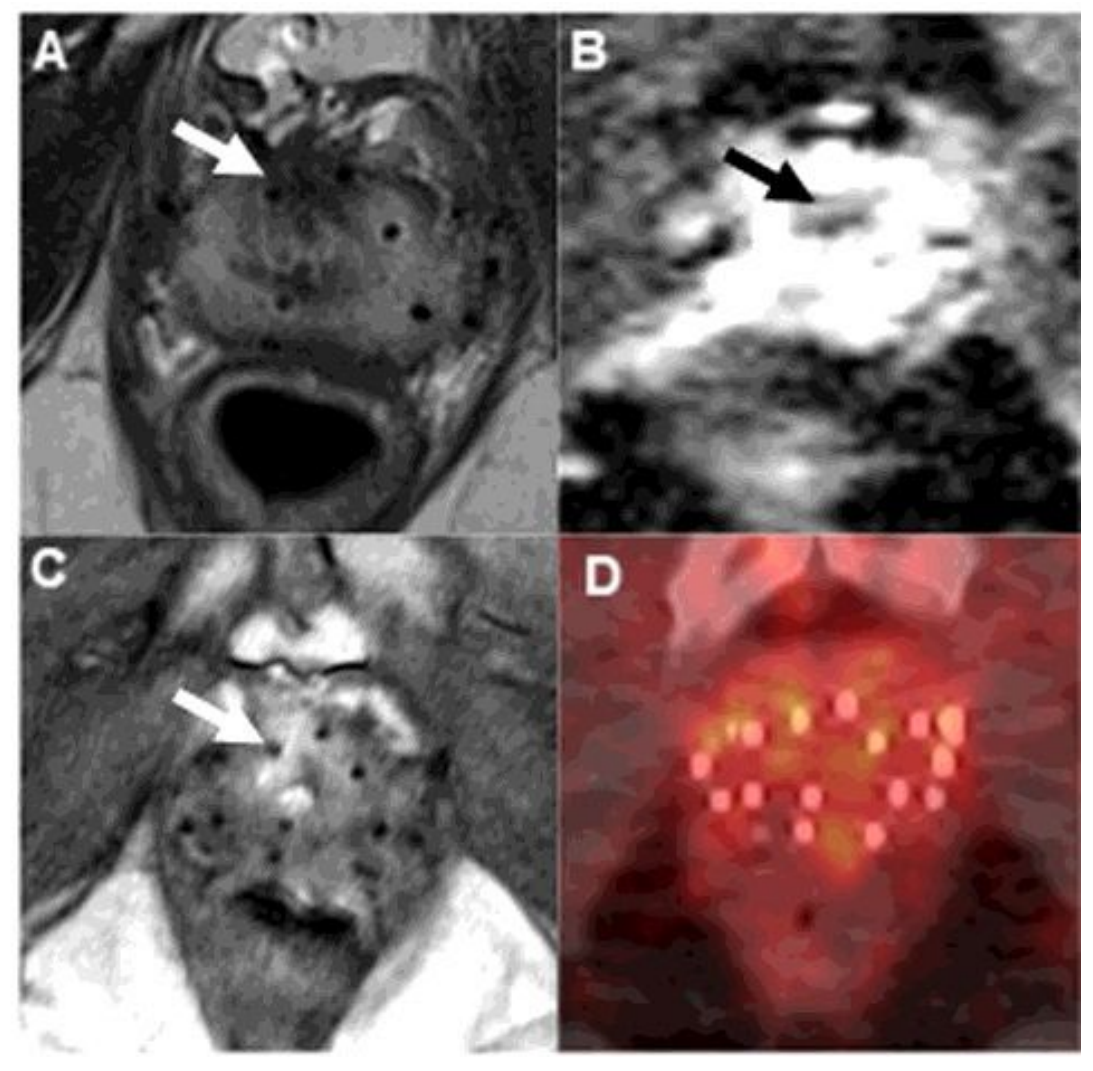

\section{Figure 3}

65-year-old patient with history of biochemical recurrence of prostate cancer 6.65 years following brachytherapy; PSA at time of imaging was $2.86 \mathrm{ng} / \mathrm{mL}$. mpMRI reveals a hypointense lesion in the midline to right apical-mid anterior transition zone (A) which is hypointense on ADC map (B) and has associated early focal enhancement on DCE imaging (C) (arrows). The prostate lesion does not demonstrate increased uptake on 18F-DCFPyL PET/CT (D). Targeted biopsy revealed benign prostatic tissue with treatment effect. 